\title{
Isquemia cerebral transitoria y riesgo de infarto cerebral isquémico
}

\author{
Luis Guillermo Rosales-Bravo
}

\section{Resumen}

La enfermedad vascular cerebral es la principal causa de hospitalización neurológica en el mundo. Costa Rica no es la excepción; en nuestros hospitales cada día es más frecuente el número de pacientes que ingresan con infartos cerebrales isquémicos. Esta común enfermedad obedece a la prevalencia de enfermedades crónicas como la hipertensión arterial, diabetes mellitus, dislipidemias, tabaquismo y la alta expectativa de vida de los costarricenses, todos considera dos factores de riesgo. Aproximadamente un $80 \%$ de los infartos cerebrales son isquémicos secundarios a la oclusión arterial aguda de un territorio vascular específico; el restante $20 \%$ corresponde a la variante hemorrágica. Las secuelas neurológicas secundarias a esta entidad son la principal causa de discapacidad crónica en los pacientes y conllevan una alta morbilidad y mortalidad.

Aunque muchos sufren el infarto cerebral isquémico en forma aguda, otro grupo de pacientes experimenta uno o varios episodios previos de isquemia cerebral transitoria, lo cual los pone en riesgo de sufrir un infarto cerebral isquémico durante un periodo corto, es decir, son pacientes vulnerables. Esta revisión pretende actualizar los conocimientos médicos, especialmente para aquellos que trabajan en la atención primaria y en los servicios de urgencias hospitalarias, que reconozcan a este grupo de pacientes y les brinden la atención médica necesaria para de prevenir un infarto cerebral isquémico. La isquemia cerebral transitoria debe ser considerada como una emergencia neurológica.

Descriptores: Isquemia cerebral transitoria, emergencia neurológica, infarto cerebral isquémico

Key words: cerebral isquemia, neurologic emergency, stroke.

Recibido: 30 de mayo de 2005

Aceptado: 8 de noviembre de 2005

Servicio de Neurología Hospital México

Abreviaturas: isquemia cerebral transitoria (ICT).

Correspondencia:

Dr. Luis Guillermo Rosales

Bravo

Apartado postal: $388-5000$

Liberia-Costa Rica

Correo electrónico:

rosalesneurologia@racsa.co.cr

ISSN 0001-6002/2006/48/1/5-11 Acta Médica Costarricense, @2006

Colegio de Médicos y Cirujanos
En los hospitales nacionales es cada vez mayor el número de pacientes que consultan al servicio de urgencias por episodios de isquemia cerebral transitoria (ICT), y pocas veces se reconoce tal entidad como una emergencia neurológica, por lo que ellos suelen ser egresados a sus hogares y, en pocos casos, referidos a la consulta externa de Neurología, para ser valorados muchos meses después del evento inicial. Una cantidad considerable de pacientes reconsultan nuevamente dentro de corto de tiempo, pero ya presentando el infarto cerebral isquémico con una alta probabilidad de complicaciones médicas intrahospitalarias, secuelas neurológicas permanentes y altos costos de hospitalización para la seguridad social.

En Costa Rica y estrechamente relacionada con la mayor longevidad de la población y otros factores de riesgo, cada vez será más frecuente esta entidad, por lo que es importante conocer sus manifestaciones clínicas, mecanismos fisiopatológicos y opciones de tratamiento 
disponibles. Existen varias modalidades diagnósticas y terapéuticas para tratar de prevenir que una ICT evolucione hasta un infarto cerebral isquémico.

En la presente revisión se analizarán la definición, causas, fisiopatología, alteraciones en los niveles celular y molecular, manifestaciones clínicas, diagnósticos diferenciales, riesgo que esta implica, la evaluación clínica por efectuar los pacientes y cómo tratar de prevenir la instauración de un infarto cerebral isquémico.

\section{Definición}

La ICT se define como episodios breves de un déficit neurológico focal, secundario a una disminución o interrupción temporal súbita del flujo sanguíneo cerebral en un territorio vascular arterial específico ${ }^{1}$; implica el concepto de reversibilidad del cuadro clínico. Desde hace muchos años, el tiempo de recuperación para este déficit neurológico se definió arbitrariamente en 24 horas, sin embargo, en la mayoría de los casos (70\%) los síntomas resuelven en menos de 20 minutos $^{2}$. Por regla general, los episodios de ICT tienen las siguientes características clínicas: son de inicio abrupto, son máximos desde el inicio, resuelven gradualmente (la mayoría antes de una hora), síntomas que se localizan según el territorio vascular afectado $\mathrm{y}$ pueden presentarse en forma repetitiva.

En aquellos pacientes en los cuales el déficit neurológico dura más de una hora, en realidad puede considerarse que ya sufrieron infartos poco extensos y que deberían ser evaluados con el propósito decidir si son candidatos para la aplicación de terapia fibrinolítica cerebral ${ }^{3}$. Lo cierto del caso es que este grupo de pacientes están expuestos a una recurrencia, la cual puede provocar un infarto cerebral isquémico de mayor dimensión que el inicial.

\section{Causas}

Tanto en la ICT como en los infartos isquémicos, los principales mecanismos que producen la interrupción del flujo sanguíneo cerebral son:

1- Embolismo arterio-arterial por el desprendimiento de un trombo plaquetario

2- Trombosis arterial secundaria a una placa ateroesclerótica con la formación de un trombo in situ.

3- Embolismo cardiaco (cardioembolismo) ${ }^{4}$.

El más común de todos los mecanismos descritos es el embolismo arterio-arterial de áreas ateroescleróticas en grandes vasos, principalmente en el arco aórtico, placas en la arteria carótida interna o en la arteria carótida común ${ }^{5}$. En segundo lugar, la formación de un trombo in situ y por último, la migración de un trombo desde las cámaras cardiacas izquierdas (aurícula o ventrículo) secundario a fibrilación auricular ${ }^{6,7} \mathrm{o}$ a la formación de un trombo intraventricular, debido a una zona de hipoquinesia ventricular por un infarto agudo al miocardio.

Otros factores que pueden producir una ICT son la hipoperfusión cerebral por estenosis arterial severa, o por un episodio hipotensión arterial sostenida. En ocasiones, una estenosis severa particularmente en los grandes vasos intracraneales o extracraneales ${ }^{8}$, puede causar compromiso hemodinámico cerebral, por lo tanto, una ICT puede ser precipitada al ponerse de pie o por cambios mínimos en la presión arterial sistémica. Otras causas menos frecuentes de ICT son: disección arterial aguda, aneurismas en el arco aórtico, estados de hipercoagulabilidad sanguínea (trombofílicos), particularmente el síndrome antifosfolípidos, uso de psicoestimulantes y displasia fibromuscular.

\section{Traducción fisiopatológica}

Ocurre un mecanismo fisiopatológico similar en la angina al miocardio, es decir, una interrupción del flujo sanguíneo cerebral, como consecuencia de la ruptura de una placa ateroesclerótica inestable, sobre la cual se formó un trombo (no oclusivo) que produjo la oclusión del lumen arterial, pero que por el tamaño del trombo y la acción del sistema fibrinolítico endógeno, fue resuelta tal y como se puede observa en la figura 1. Se puede comparar la ICT con un "angor cerebral", en el sentido de que se altera

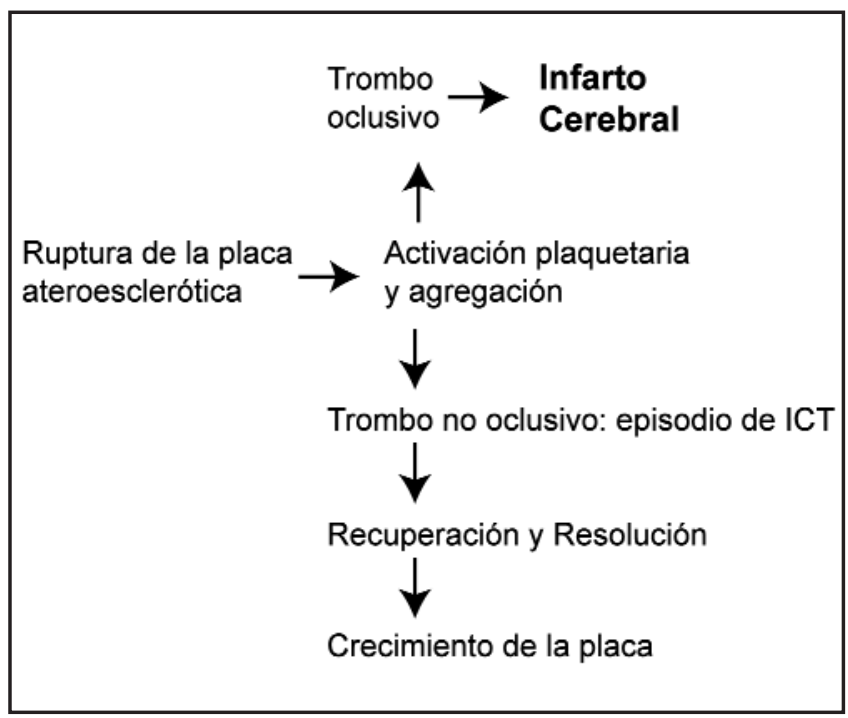

Figura 1. Mecanismo fisiopatológico por medio del cual se producen los episodios de ICT y los infartos cerebrales isquémicos. Si se forma un trombo oclusivo se producirá un infarto cerebral isquémico. Si se forma un trombo no oclusivo ocurrirá un episodio de ICT. 
transitoriamente el flujo sanguíneo cerebral en un área específica y aunque no suele manifestarse con dolor, se producen manifestaciones neurológicas características, como se describirá más adelante. Si el trombo ocluye por completo el lumen arterial, se producirá invariablemente el infarto cerebral isquémico ${ }^{9}$ (figura 2). Por lo anterior, la ICT debe ser considerada una emergencia neurológica.

Es durante un episodio de ICT se producen varias alteraciones a nivel celulares y moleculares que deben ser mencionadas. El flujo cerebral normal es de $0,5 \mathrm{cc} / \mathrm{g}$ de cerebro/min, y cuando ocurre una disminución del flujo sanguíneo cerebral (alrededor de $0,35 \mathrm{cc} / \mathrm{g}$ de cerebro/min.), se pierde la homeostasis celular normal, y se altera el metabolismo energético ${ }^{10}$. Lo anterior provoca que se inicie el metabolismo anaerobio de la glucosa, llevando a la producción de ácido láctico y la eventual acidosis tisular. Además, la hipoxia transitoria provoca desacoplamiento de la fosforilación oxidativa, lo cual disminuye la producción de adenosina trifostato $\mathrm{y}$ otros nucleótidos energéticos indispensables para la neurona ${ }^{11}$. Algunos canales de membrana neuronal necesitan del adenosina trifostato para mantener la homeostasis iónica en contra del gradiente de concentración, por lo tanto, se produce salida de potasio del interior celular hacia el espacio intersticial y desplazamiento del sodio hacia el citoplasma neuronal, acarreando agua. Esto provoca edema intracelular (citotóxico) e incapacidad de mantener el potencial de membrana, lo cual origina fallo en la despolarización de la célula y suprime la actividad eléctrica neuronal, principalmente por la pérdida de la función de las bombas de intercambio iónico en el nivel de la membrana celular ${ }^{12}$. Tales procesos afectan los diferentes grupos neuronales y causan pérdida de sus funciones.

La disrupción transitoria neuronal provoca alteraciones en la transmisión de los impulsos nerviosos normales y, dependiendo del territorio cerebral afectado, se manifestará clínicamente como un déficit neurológico transitorio (motor, sensitivo, visual). Estas alteraciones suelen reinvertirse cuando se reestablece el flujo cerebral, dado que el tiempo durante el cual hubo hipoperfusión fue corto. Si el proceso no se revierte se producirá daño mitocondrial, acumulación de calcio intracelular, activación de leucocitos con liberación de mediadores inflamatorios, generación de radicales libres y liberación de toxinas exitatorias, todo lo cual causará infarto cerebral isquémico ${ }^{13}$.

\section{Manifestaciones clínicas}

Muchos de los pacientes consultan al servicio de emergencias por síntomas sensitivos, motores, cerebelosos, visuales o combinación de estos, lo cual se traduce en una disfunción cerebral, cerebelar, tallo cerebral o de la retina ${ }^{14}$. Las manifestaciones clínicas dependen del territorio vascular afectado y son muy variadas, pero provocan debilidad,

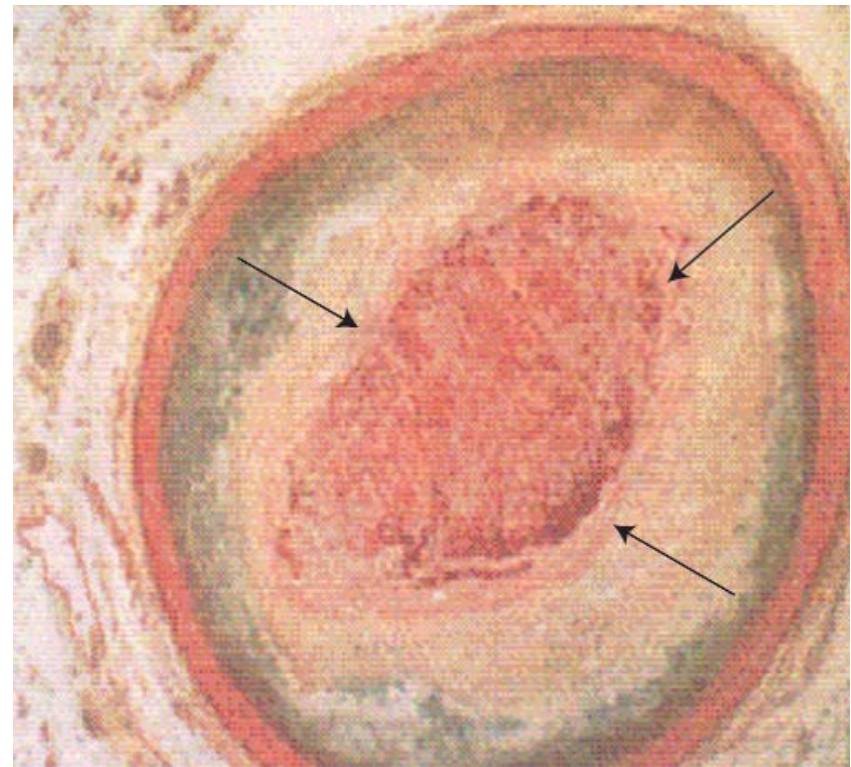

Figura 2. La formación de un trombo intraarterial (flechas negras) en la circulación cerebral, es la responsable de producir un infarto cerebral isquémico. Muchos de estos pacientes experimentan episodios previos de ICT antes de que se produzca el infarto. La ilustración corresponde a trombo en la porción proximal de la arteria cerebral media derecha. (Cortesía del Dr. Raúl Bonilla. Servicio de Patología. Hospital San Juan de Dios).

adormecimiento, incoordinación o trastornos visuales transitorios ${ }^{15}$. Se puede hacer una división general de los síntomas de acuerdo con la interrupción del flujo cerebral, según ocurra en la circulación cerebral anterior o en la posterior:

\section{a- Circulación anterior (distribución carotídea)}

Amaurosis fugax: episodio de ceguera monocular transitoria ipsilateral, no dolorosa, secundaria a isquemia en el territorio de la arteria retiniana ${ }^{16}$, o más comúnmente con síntomas visuales diversos, tales como visión borrosa, escotomas, percepción de destellos luminosos, entre otros. Se debe tener presente que la arteria retiniana es rama directa de la carótida interna. No todos los trastornos visuales transitorios son consecuencia de isquemia retiniana, como se muestra en el cuadro 1.

Disfunción de un hemisferio cerebral: debilidad, sensación de adormecimiento o de pesantez, o parestesias contralaterales al territorio vascular afectado (mano y brazo, mano y cara, hemicuerpo), disartria y afasia motora, receptiva.

\section{B- Circulación posterior (distribución vertebrobasi- lar)}

Pérdida visual binocular (en uno o ambos campos visuales), diplopia, vértigo, ataxia, disartria, disfagia, pérdida auditiva, pérdida del tono muscular, debilidad, parestesias, sensación de pesantez, sensación de adormecimiento unilateral o bilateral. Los síntomas pueden alternar 


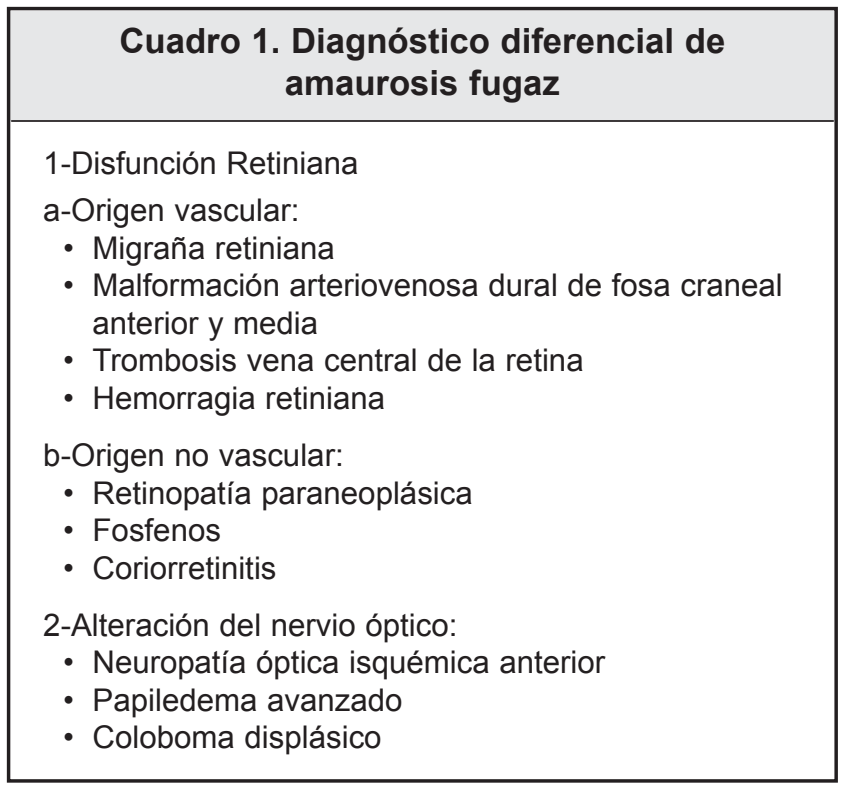

entre el lado izquierdo y derecho en diferentes episodios de ICT y pueden ser cruzados sobre ambos.

\section{Diagnósticos diferenciales}

No todo déficit neurológico súbito es secundario a una ICT, por lo tanto, se deben descartar otras entidades neurológicas productoras de déficits transitorios, como se muestra en el cuadro 2. Algunos síntomas neurológicos, por su forma y patrón de presentación, hacen poco probable que se trate de una ICT, por lo que es importante tomarlos en consideración, tal y como se refiere en el cuadro 3.

\section{Riesgo de un eventual infarto isquémico cerebral}

La ICT es un potente predictor de infarto cerebral isquémico, con mayor riesgo durante los primeros días y semanas. En un estudio se demostró que durante los 90 días posteriores a la ICT, uno de cada 9 pacientes sufrieron infarto isquémico, la mitad de ellos en los primeros 2 días ${ }^{17}$. El riesgo para los pacientes con fibrilación auricular fue tan alto como el 11\%. Similarmente, en un grupo de 187 pacientes el riesgo de sufrir un infarto isquémico durante el primer mes posterior a una ICT fue del $4 \%{ }^{18}$. En aquellos pacientes con estenosis sintomática de la arteria carótida interna que presentaron episodios de ICT, el riesgo de sufrir un infarto isquémico durante los siguientes 90 días fue tan alto como el $20 \%{ }^{19}$. Por lo anterior, debe entenderse como un síntoma de alerta que se traduce en una enfermedad vascular que se torna inestable y debe abordarse como una emergencia neurológica.

\section{Cuadro 2. Diagnósticos diferenciales de} isquemia ICT

- Migraña complicada

- Convulsiones focales/ parálisis de Todd

- Síncope/presíncope

- Alteraciones metabólicas (hipoglicemia, hiperglicemia, hipercalcemia, hiponatremia)

- Amnesia global transitoria

- Lesiones estructurales intracraneales:

- Tumor cerebral (meningioma)

- Hematoma subdural crónico

- Aneurisma gigante

- Malformación arteriovenosa

- Brote desmielinizante (esclerosis múltiple)

- Cataplexia

Cuadro 3. Síntomas que van en contra de un episodio de ICT

- Síntomas cerebrales globales: confusión, pérdida de la conciencia, presíncope, síncope.

- Episodios de disfunción neurológica que duran pocos segundos

- Síntomas neurológicos que marchan o migran de una parte del cuerpo a otra o que son fluctuantes en el tiempo.

- Síntomas neurológicos "positivos": movimientos involuntarios, sacudidas bruscas.

- Vértigo solo. El vértigo isquémico usualmente se acompaña de otros síntomas neurológicos (ataxia, nistagmus).

\section{Evaluación clínica y prevención del infarto cerebral}

Tras realizarse una detallada historia clínica y examen físico del paciente en el Servicio de Emergencias, y considerar que este sufrió un episodio de ICT, se debe referir o ingresar al Servicio de Neurología para una evaluación clínica más cuidadosa, con la finalidad de iniciar las medidas terapéuticas para prevenir un eventual infarto cerebral isquémico. Uno de los primeros estudios por efectuar es una tomografía axial computarizada de cerebro, infratentorial y supratentorial, sin medio de contraste, para descartar lesiones estructurales intracraneales que pueden simular una ICT. Lo usual es que tal estudio sea normal en este grupo de pacientes. En urgencias también se pueden practicar otras pruebas de laboratorio y gabinete: electrocardiograma, radiografía simple de tórax, hemograma completo, 
tiempos de coagulación y análisis bioquímico completo. Una vez hospitalizado, se deben completar otros estudios bioquímicos, hematológicos y de neuroimágenes, para iniciar las medidas terapéuticas correctivas y preventivas de un eventual infarto cerebral isquémico. Los principales estudios de laboratorio y gabinete que se deben practicar a estos pacientes se muestran en el cuadro 4 .

En la mayoría de estos pacientes se demuestran varias alteraciones bioquímicas y de la circulación cerebral que los exponen a sufrir un infarto isquémico, muchas de las cuales pueden ser corregidas o aminoradas. Una de las fundamentales es la enfermedad ateroesclerótica en la circulación cerebral, la cual se encuentra en relación directa con la hipertensión arterial, diabetes mellitus, tabaquismo y dislipidemia ${ }^{20}$. Actualmente se utilizan varios medicamentos antitrombóticos para la prevención de infartos cerebrales. El uso diario de aspirina de $100 \mathrm{mg} / \mathrm{d}$ vía oral, está claramente demostrado que previene infartos cerebrales ${ }^{21}$; a los pacientes que no toleran este medicamento, se les puede prescribir clopidogrel de $75 \mathrm{mg} / \mathrm{d}$, vía oral ${ }^{22}$; también se pueden combinar ambos fármacos para producir un efecto sinérgico.

Un grupo especial de pacientes son aquellos en los cuales se documenta la fibrilación auricular con un trombo intracavitario, quienes han sufrido episodios previos de ICT o un infarto isquémico cerebral, pacientes con estenosis sintomática de la arteria carótida interna (mayor del 50\%) y pacientes con placas inestables en el arco aórtico demostradas por ecocardiograma transtorácico; dado que están en alto riesgo de recurrencia, se utiliza la anticoagulación sistémica con warfarina vía oral, manteniendo un INR (International Normalized Ratio) entre 2 y $3{ }^{23}$. La utilización de warfarina en adultos mayores se debe hacer con extrema precaución, tomando en cuenta la relación riesgo/beneficio, para evitar la sobreanticoagulación y los riesgos que conlleva ${ }^{24}$. En adultos mayores con síndromes demenciales, incoordinación para la marcha, posibilidad de interacciones medicamentosas de la warfarina con los múltiples fármacos utilizados por ellos, se recomienda mejor no usarla y, en su lugar, prescribir aspirina.

La presencia de hipercolesterolemia se vincula con el desarrollo temprano de ateroesclerosis ${ }^{25}$, por lo que los pacientes deben ingerir dietas bajas en contenido de lípidos. La utilización de estatinas (ej. lovastatina $20-40 \mathrm{mg} / \mathrm{d}$ vía oral, pravastatina $40 \mathrm{mg} / \mathrm{d}$ vía oral), fármacos que por su acción bloqueadora de la enzima 3-hidroxi-3-metilglutaril coenzima A reductasa, ayudan a la disminución de los niveles séricos de triglicéridos y colesterol implicados en la génesis de la placa ateromatosa ${ }^{26,27}$. Además de sus acciones hipolipemiantes, las estatinas poseen otras propiedades farmacológicas, tales como acción estabilizadora y antiinflamatoria sobre las placas ateroescleróticas, propiedades que en conjunto se conocen como pleiotrópicas, por lo que se recomienda su uso como medicamento preventivo de eventos cardiovasculares.

\begin{tabular}{l}
$\begin{array}{c}\text { Cuadro 4. Principales estudios de laboratorio y } \\
\text { gabinete en pacientes con ICT }\end{array}$ \\
\hline - Hemograma completo \\
- Tiempos de Coagulación/ Fibrinógeno \\
- Glicemia \\
- Perfil lipídico ( Colesterol total, HDL, LDL. \\
Triglicéridos) \\
- Homocisteinemia \\
- Niveles de folatos y vitamina B12 \\
- VDRL \\
- Radiografía simple de Tórax \\
- Electrocardiograma \\
- Ecocardiograma transesofágico \\
- Ultrasonido dupplex de arterias carótidas y arterias \\
- vertebrales \\
- TAppler transcraneal \\
- Arteriografía cerebral \\
(para casos seleccionados)
\end{tabular}

Otra modalidad terapéutica para pacientes con estenosis mayores del $50 \%$ en la arteria carótida interna es la endarterectomía carotídea ${ }^{28}$, la cual se reserva para aquellos casos en los cuales no existen contraindiciones médicas que aumenten su morbimortalidad. Este procedimiento quirúrgico debe ser practicado por cirujanos vasculares experimentados; el porcentaje de morbilidad es aproximadamente del $3 \%$.

Desde hace varios años se realiza arteriografías cerebrales diagnósticas ${ }^{29}$ para pacientes en los cuales, por medio del ultrasonido dupplex o el doppler transcraneal, se demostró alteraciones en el flujo cerebral, lo que sugiere una enfermedad ateroesclerótica significativa en los vasos arteriales intracraneales. Con esta modalidad diagnóstica es posible visualizar mejor el lecho vascular cerebral y determinar la presencia de estenosis sintomáticas en las arterias carótidas comunes, carótidas internas y sus ramas intracraneales. En casos seleccionados se puede prevenir la oclusión arterial mediante la colocación de prótesis endovasculares (stent) las cuales restablecen el flujo cerebral, como se ilustra en el caso clínico de la figura 3. Este procedimiento puede ser efectuado por neurocirujanos endovasculares, neurorradiólogos o neurólogos vasculares con el entrenamiento apropiado ${ }^{30}$. Es preciso utilizar dispositivos de protección cerebral durante el procedimiento, para evitar la migración de restos de placas ateroescleróticas y detritus celulares que pueden producir microinfartos isquémicos; si este procedimiento es más efectivo que la endarterectomía carotídea, es aún tema de discusión y análisis ${ }^{31,32}$. 

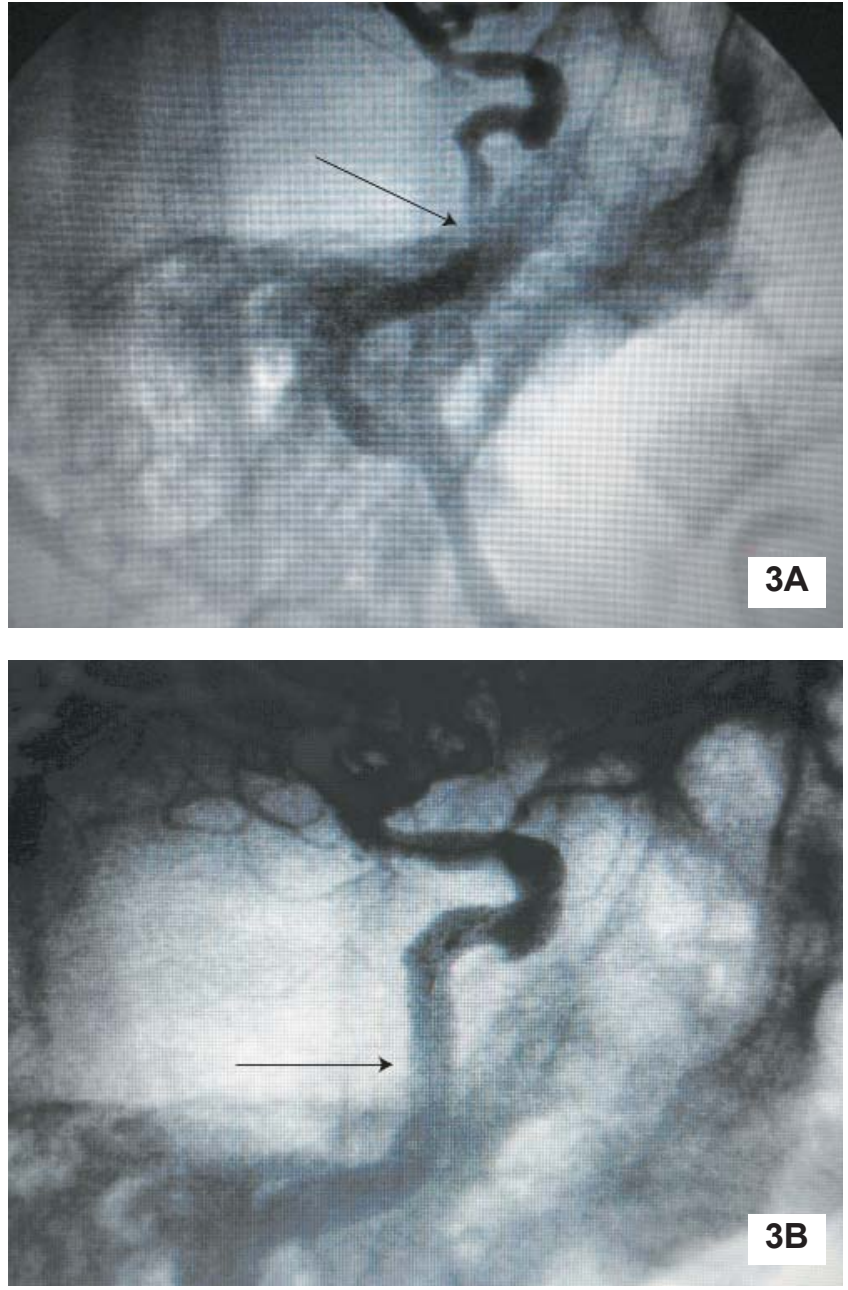

Figura 3. Arteriografía cerebral. A) proyección lateral derecha en la cual se observa una estenosis significativa $(50 \%)$ de la porción cavernosa de la arteria carótida interna derecha (flechas). B) resolución de la estenosis posterior a la colocación de una prótesis endovascular (stent) (flechas). Se trataba de un paciente masculino de 59 años quien fue ingresado al servicio de Neurología por historia de varios episodios previos de amaurosis fugax en ojo derecho, hemiparesia y parestesias en hemicuerpo izquierdo (episodios de ICT). (Cortesía del Dr. Roger Torres Cordero, Neurocirujano Endovascular. Servicio de Neurocirugía. Hospital San Juan de Dios.)

Importante, además de lo mencionado, es el adecuado control de la presión arterial sistémica en los pacientes hipertensos, lo mismo que de los niveles de glicemia y hemoglobina glicosilada, el abandono del hábito de fumar y la practica de ejercicio físico regular ${ }^{33}$. Todas estas medidas previenen la aparición de la enfermedad vascular cerebral.

En nuestro país es de suma relevancia educar a la población general, mediante campañas informativas en la atención médica primaria y en los diferentes medios de comunicación, acerca de cómo reconocer tempranamente los síntomas de una ICT o de un infarto cerebral, para que busquen atención médica rápida y eviten que se produzca un infarto cerebral extenso, con posibles secuelas neurológicas permanentes (figura 4 ).
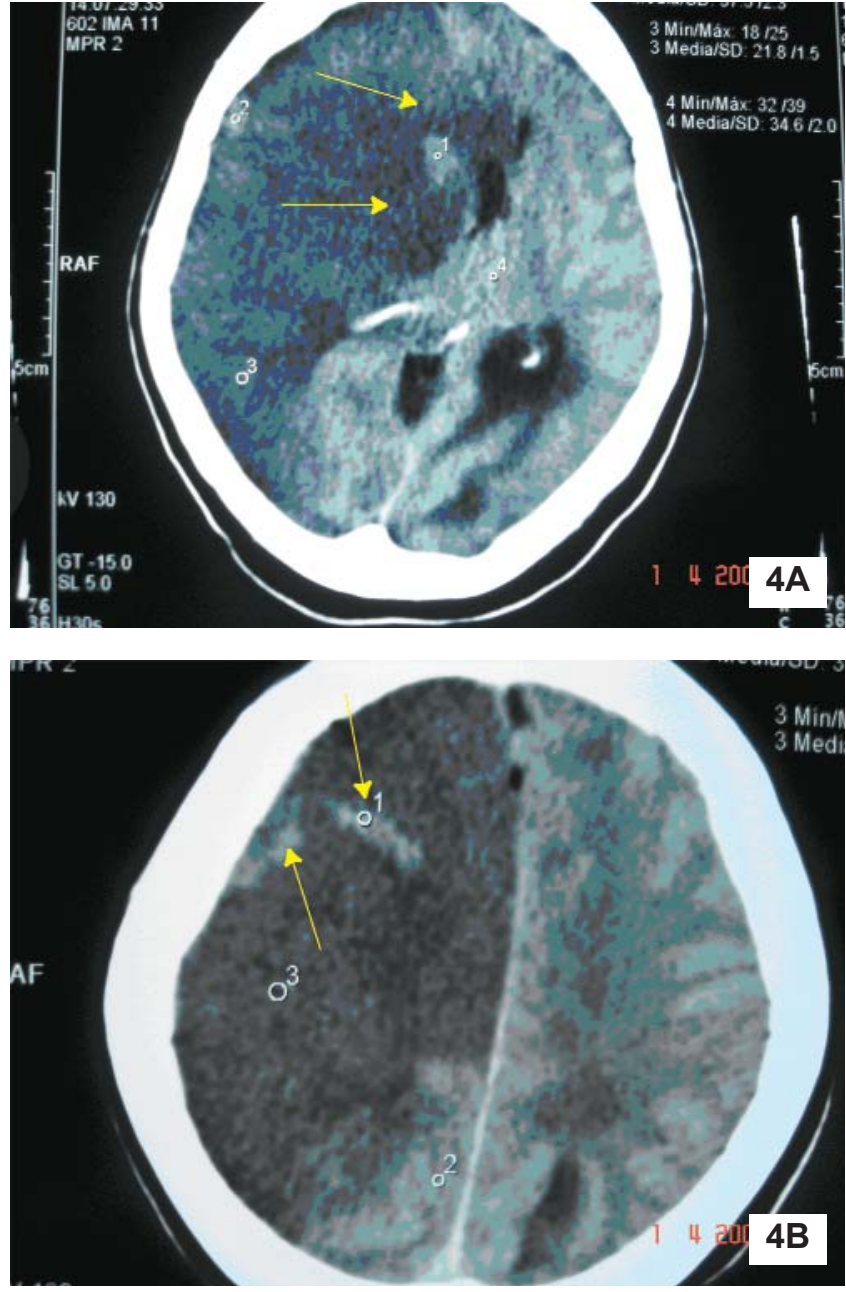

Figura 4. A) Tomografía axial computarizada helicoidal de cerebro la cual muestra un infarto cerebral isquémico. Se observa una zona de hipoatenuación extensa en la hemisferio cerebral derecho, la cual produce efecto de masa y desplazamiento de la línea media contralateralmente con colapso de los cuernos anteriores de los ventrículos laterales (flechas).B) Dentro de la zona de hipoatenuación se identifican pequeñas zonas no confluentes de sangrado, lo cual corresponde a una transformación hemorrágica espontánea del infarto isquémico (flechas). Se trataba de una paciente femenina de 72 años quien había consultado al menos en 2 ocasiones al servicio de urgencias por episodios de ICT. (Dr. Luis Guillermo Rosales Bravo. Servicio de Neurología. Hospital México).

\section{Abstract}

Stroke is the first cause of neurological hospitalization in the world. Costa Rica is not the exception, in our hospitals the amount of patients who are admitted with ischemic stroke increases every day. This condition is more common due to the high prevalence of chronic diseases, such as hypertension, diabetes mellitus, and dyslipidemia, besides smoking and the high costarrican life expectancy, are all considered risk factors for development of a stroke. Approximately $80 \%$ of the strokes are ischemic in nature 
secondary to an acute arterial occlusion of an specific vascular territory, the remaining $20 \%$ are of hemorrhagic origin. The neurological impairment secondary to strokes, in these patients is the main cause of chronic disability and is related with high morbility and mortality.

Although many individuals have an acute ischemic stroke, other patients will have 1 or more events of transient ischemic attacks, indicating that they are at risk for an ischemic stroke in a brief period of time. This article reviews the current medical knowledge, and is written especially for general practitioners and emergency physicians, so they can recognize the patients at risk and prevent them from having an ischemic stroke. Transient ischemic attacks must be considered a neurological emergency.

\section{Referencias}

1. Claiborne S.Transient Ischemic Attack. N Engl J Med 2002; 21:1687-1692.

2. Johnson RT, Griftin JW and Mcarthur JC. Current therapy in Neurologic disease. Sixth edition. USA: Mosby, 2002.

3. The National Institute of Neurological Disorder and Stroke rt-PA Stroke Study Group. Tissue plasminogen activator for acute ischemic stroke. N Engl J Med 1995;333:1581-1587.

4. Bricker ME, MD. Cardioembolic Stroke. Am J Med 1996;100:465474.

5. Hankey G, Slattery J, Warlow C. Transient ischaemic attacks: which patients are at high (and low) risk of serious vascular events? J Neurol Neurosurg Psychiatry 1992;55:640-652.

6. Wolf P, Abbot R, Kannel W. Atrial fibrillation as an independent risk factor for stroke: the Framingham Study. Stroke 1991;22:983-988.

7. Wolf P, Mitchell J, Baker C, Kannel W, D`Angostino R. Impact of atrial fibrillation on mortality, stroke, and medical costs. Arch Intern Med 1998;158:229-234.

8. Norris J. Risk of cerebral infarction, myocardial infarction and vascular death in patients with asymptomatic carotid disease, transient ischemic attack and stroke. Cerebrovasc Dis 1992;2(suppl):2-5.

9. Adams HP. Treating ischemic stroke as an emergency. Arch Neurol.1998;55:457-461.

10. Barinagarrementería F, Cantú C. Enfermedad Vascular Cerebral. México: McGraw- Hill Interamericana, 1999.

11. Lee J, Zipfel G, Choi D. The changing landscape of ischaemic brain injury mechanism. Nature 1999;399:Suppl:A7-A14.

12. Brott T, Bogousslovsky J. Treatment of acute ischemic stroke. N Eng J Med 2000;343:710-722.

13. Kristian T, Siesjo B. Calcium in ischemic cell death. Stroke 1998;29:705-718.

14. Guidelines for the management of transient ischemic attacks. From the Ad Hoc Committee on Guidelines of the Management of Transient Ischemic Attacks of the Stroke Council of the American Heart Association. Stroke 1994;25:1320-1335.

15. Adams H Jr, del Zoppo G, von Kummer R. Management of stroke: a practical guide for the prevention, evaluation and treatment of acute stroke. USA: Professional Communications NC, 2002.

16. Donders R, Kappelle L, Derksen R. Transient monocular blindness and antiphospholipid antibodies in systemic lupus erythematosus. Neurology 1998;51:535-540.
17. Johnston S, Gress D, Browner W, Sidney S. Short-term prognosis alter emergency department diagnosis of TIA. JAMA 2000;284:2901-2906.

18. Dennis M, Bamford J, Sandercock P, Warlow C. Prognosis of transient ischemic attacks in the Oxfordshire Community Stroke Project. Stroke 1990;21:848-853.

19. Streifler J, Eliasziw M, Benavente O. The risk of stroke in patients with first-ever retinal vs. hemispheric transient ischemic attacks and high-grade carotid stenosis: North American Symptomatic Carotid Endarterectomy Trial. Arch Neurol 1995;52:246-249.

20. O'Leary D, Polak J, Kronmal R. Carotid-artery intima and media thickness as a risk factor for myocardial infarction and stroke in older adults. Cardiovascular Health Study Collaborative Reserch Group. N Eng J Med 1999;340:14-22.

21. Hart R, Palacio S, Pearce L. Atrial fibrillation, stroke and acute antithrombotic therapy. Analysis of randomized clinical trials. Stroke 2002;33:2722-2727.

22. Paciaroni M, Bogousslovsky J. Clopidogrel for cerebrovascular prevention. Cerebrovasc Dis 1999;9:253-260.

23. Adams H. Emergent use of anticoagulation for treatment of patients with ischemic stroke. Stroke 2002;33:856-861.

24. Tanne D, Turgerman D, Adler Y. Management of acute ischemic stroke in the elderly. Drugs 2001;32:1074-1078.

25. Hachinski V, Graffagnino C, Beaudry M. Lipids and Stroke: a paradox resolved. Arch Neurol 1996;53:303-308.

26. Plehn J, Davis B, Sacks F. Reduction of stroke incidence after myocardial infarction with pravastatina: the Cholesterol and Recurrent Events (CARE) Study. The care investigators. Circulation 1999;99:216-223.

27. Blauw G, Lagaay A, Smelt A, Westendorp R. Stroke, statins, and cholesterol. A meta-analysis of randomized, placebo-controlled, doubleblind trials with HMG-CoA reductasa inhibitors. Stroke 1997;28:946-950.

28. Biller J, Feinberg W, Castaldo J. Guidelines for carotid endarterectomy: a statement for healthcare professionals from a Special Writing Group of the Stroke Council, American Heart Association. Circulation 1998;97:501-509.

29. Sacks D, Connors J. Carotid Stent Placement, Stroke Prevention, and Training. Radiology 2004;234:49-52.

30. Connors J, Saks D, Furlan A, Selman W, Russell E, Stieg P et al. Training, Competency, and Credentialing Standards for Diagnostic Cervicocerebral Angiography, Carotid Stenting, and Cerebrovascular Intervention : A Joint Statement from the American Academy of Neurology, American Association of Neurological Surgeons, American Society of Interventional and Therapeutic Radiology, American Society of Neuroradiology, Congress of Neurological Surgeons, AANS/CNS Cerebrovascular Section, and Society of Interventional Radiology. Radiology 2005;234:26-34.

31. Yadav J, Wholey M, Kuntz R, Fayad P, Katzen B, Mishkel G et al. Protected Carotid-Artery Stenting versus Endarterectomy in HighRisk Patients. N Eng J Med 2004;351:1493-1501

32. Lin P, Bush R, Lubbe D, Cox M, Zhou W, McCoy S et al. Carotid artery stenting with routine cerebral protection in high-risk patients. Am J Surg 2004;188:644-652.

33. Solenski N. Transient ischemic attacks: Part II. Treatment. Am Fam Physician 2004;69:1681-1688. 